\title{
Visual Analysis of Cognitive Structure based on the Human-Machine Interaction
}

\author{
Cheng Rengui ${ }^{1}$, Liu Changyong ${ }^{1}$ and Meng Shimin ${ }^{1,2 *}$ \\ ${ }^{1}$ Graphical Image Lab, Wuyi University, Wuyishan 354300, Fujian, China; ${ }^{2}$ DNAgent Lab@UlinkM.com INC., Wuyis- \\ han 354300, Fujian, China
}

\begin{abstract}
Learning is a process that changes the cognitive structure, which is the important representation of advanced cognition. Due to the difficulties in observing cognition, the empirical and visual researches of cognitive structure are significant. To realize the empirical research, the first issue is how to observe the cognitive structures. Based on the humanmachine interaction, we have established a new "Brain cognitive body - cognitive coupling Situations - information Manifolds" (BSM) cognition observation platform. This platform allows the users to observe the cognitive processes and structures, collect and re-structure the cognitive activity information. Additionally, the cognitive structure imaging, the empirical and visual analysis and the dimension reduction for high-dimensional cognitive manifolds can be realized on this BSM platform. Here, we have selected the tree-based network to represent the low-dimensional cognitive manifolds to develop a visual and vectorial model of layered cognitive structure. The digital model of cognitive structure is able to provide the basis for studying the empirical research, mathematics and dynamics of the cognitive structure, and the new ways for the advanced cognitive research.
\end{abstract}

Keywords: Cognitive structure, complete network, human-machine interaction, topological manifold, visual analysis.

\section{INTRODUCTION}

Learning is a process of cognitive structure and intelligence developments. The cognitive structure is an important concept for studying the advanced cognitive activity. However, the equation, mechanism, objectivity and empirical research of cognitive structure are still at the preliminary stage. Since 2011, we have investigated the human-machine interaction from the perspective of cognition observation, and try to develop a novel theory and method for cognitive structure observation $[1,2]$. First, the cognition takes place in the interaction and coupling processes of "brain cognition body - environments outside the body", and the cognitive activities in the brain are coupled to the observed thoughts outside the brain based on advanced cognitive philosophy such as situated cognition, embodied cognition, distributed cognition and extended cognition [3-5], which is called "cognitive coupling theory and thoughts of cognitive coupling observation". Secondly, the cognitive morphisms exsits in human-machine interaction environment based on the category theory and cognitive coupling states. The environment can be divided into "Brain cognition body - cognitive coupling Situations - information Manifolds" [6] (BSM), which is called "cognitive manifold observation platform". We have demonstrated that the principle of natural isomorphism and coupling transform existed between the cognitive body and information manifolds in BSM. This principle ensures that the collected data in the coupling states of human-

\footnotetext{
*Address correspondence to this author at the Graphical Image Lab, Wuyi University, Wuyishan 354300, Fujian, China and DNAgent Lab@UlinkM. com INC., Wuyishan 354300, Fujian, China; Tel: (0086)18960624795; Fax: (0086)599-5136186; E-mails: crg@wuyiu.edu.cn, mengshimin@ulinkm.com
}

machine interaction is objective and effective. In the brain, the cognitive structures are isomorphically mapped to the outside information manifolds; the covariance exists between the cognitive processes of brain cognitive bodies and the cognitive orbits in information manifolds, which makes the cognitive modulation possible.

The human-machine interaction environment allows us to collect more accurate cognitive information and create the digital environment which can be modulated $[7,8]$. It can also provide accurate laboratories for studying the cognitive dynamics $[9,10]$. For example, the exploration of cognitive theory requires advanced tools and platforms like "cloud chamber" and "accelerator" to study the microscopic particles in high energy physics.

Fig. (1) shows that the cognitive manifolds in the brain are mapped to the information manifolds by the coupling situation of human-machine interaction, which can be named as "cognitive high-dimensional manifold observation method in cognitive coupling states of man-machine interaction".

With the cognitive observation tool, method and platform, we will further explore the secrets of cognitive structures, and investigate the re-structuring issue of observed cognitive data: the elements, objects, relationship and measurement standard of cognitive structures can be determined from the perspective of mathematics and empirical research $[11,12]$. What topology and metric space will be chosen to re-structure the observed cognitive information from BSM?

From the perspective of the manifold, the cognition restructuring is an issue of mapping high-dimensional cognitive manifolds to a low-dimensional space based on the BSM observation platform. Thus, a simple and efficient structure 


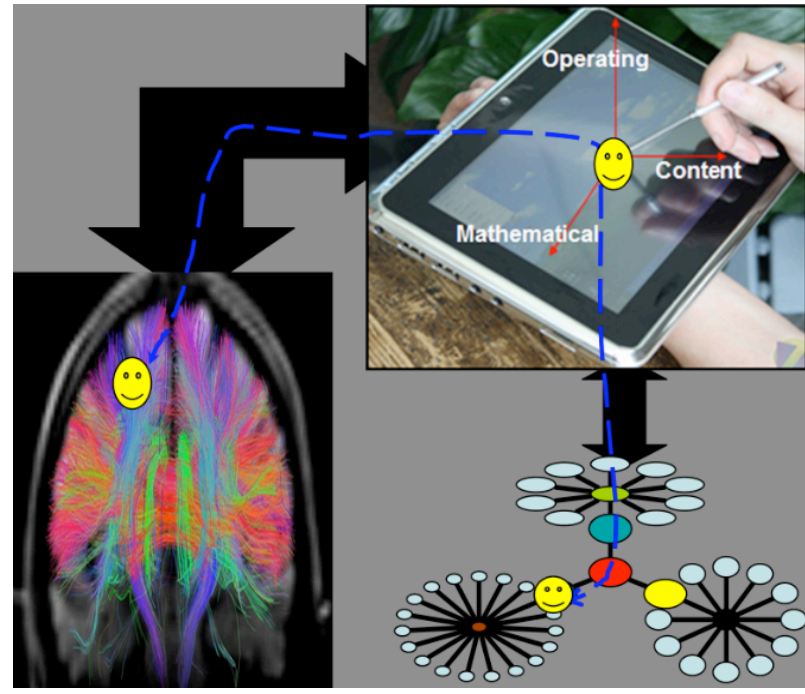

Fig. (1). BSM and cognitive high-dimensional manifold observation method in cognitive coupling states of man-machine interaction.

of cognitive low-dimensional manifold should be selected, which can represent the objects and relationships in highdimensional spaces by computers.

When the structure of low-dimensional manifolds was selected to represent high-dimensional cognitive manifolds, it should be considered: (1) the structure of low-dimensional cognitive manifolds should be effective to contain the necessary information in the high-dimensional cognitive manifolds; (2) the structure of low-dimensional cognitive manifolds can be empirically validated as the physics and chemistry, and the cognitive data can be collected on the BSM platform; (3) the structure of low-dimensional cognitive manifolds can be digitized and processed by computers, and represent the cognitive mathematical equations and the processes of cognitive dynamics.

Here, the graphs were selected to represent the lowdimensional mapping structures for the high-dimensional cognitive manifolds, and the tree-based network was used to describe the structure of cognitive topology. A metric space was also created by introducing the related metrics. Except the consideration from the perspective of mathematics, we also introduced the concrete concepts in the fields of cognition and learning for comprehensive analysis.

\section{ARCHITECTURE AND FUNTIONALITY OF COG- NITIVE STRUCTURE OBSERVATION PLATFORM}

Category theory [13], as well as manifold and topology theory, are introduced in our approach. When the cognitive processes are observed in the brain, we assume that the cognition is in a high-dimensional cognitive manifold. According to the manifold analysis theory, we should map a local structure of a high-dimensional manifold to a lowdimensional manifold in a metric space (Fig. 2).

What is the local structure of a high-dimensional manifold? How do we obtain the content of the local structure? How do we collect the cognitive objects and data in cognitive processes after receiving the content of the local structure? What structures do the received data re-structure on the

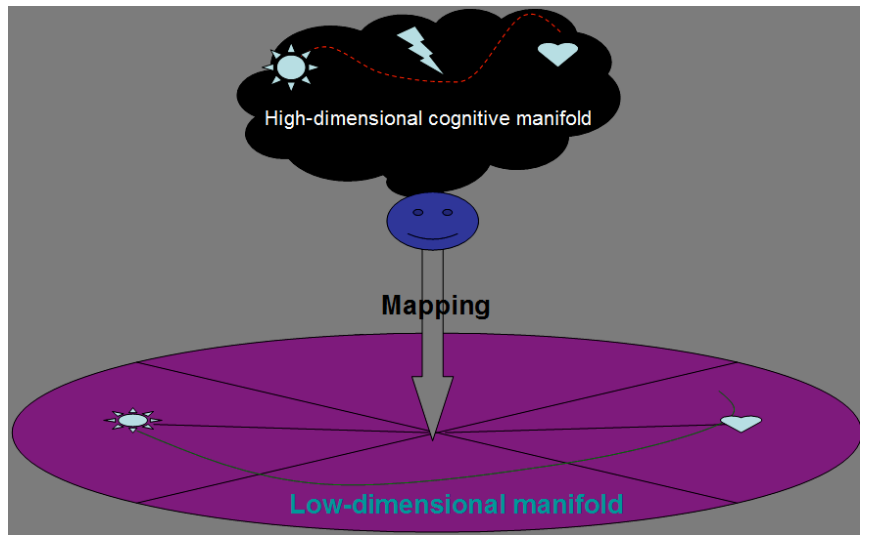

Fig. (2). The architecture of BSM observation platform - a local structure of a high dimensional manifold is mapped to a lowdimensional cognitive manifold.

low-dimensional manifold? All these questions are required to be answered in the cognitive activity observation in BSM.

\subsection{Coupling Situations in BSM Architecture}

Different with the BSM platform, the traditional psychology studies collect data through face-to-face interviews or questionnaires. Recently, the computers and BCI devices are employed. For BSM platform, the human-machine interaction systems are the basic tools for collecting the users' cognitive information. Undoubtedly, the cognitive coupling situation is a critical part.

The cognition observation in BSM is through the indirect observation in human-machine cognitive coupling situations. For the advanced cognitive philosophy, the interfaces of human-machine cognitive situations represent the cognition in the brain in the process of human-machine interaction.

When the human-machine interaction is in cognitive coupling states, we believe that the cognition in the brain is coupled with the cognitive situation, which is similar with the quantum entanglement as a fusion of cognition-situation. Thus, the observation of cognitive situations is identical to that of the cognition in the brain (Fig. 3).

Undoubtedly, the design of human-machine coupling situations is critical to the observation in BSM. The following issues are needed to satisfy the coupling situations: (1) the content and operations in cognitive situations should match the cognitive ability of observers;(2)the observed person can understand the cognitive coupling situations;(3)the observed person can express their own cognitive content and processes through the human-machine interaction;(4)the operations performed by the observed person in cognitive situations can be recorded, collected and transformed to the symbol sequences;(5) it is assumed that the observed person is reliable, and there is no irresponsible actions during the observation [1].

\subsection{Analysis of High-dimensional Cognitive Manifolds in BSM}

In BSM,(1)M denotes a high-dimensional cognitive manifold, and $\varphi$ denotes a homeomorphic mapping of a local structure in $\mathrm{M}$ to (2) a Euclid space, (3) o denotes a cognitive process and orbit in M (Fig. 4). 


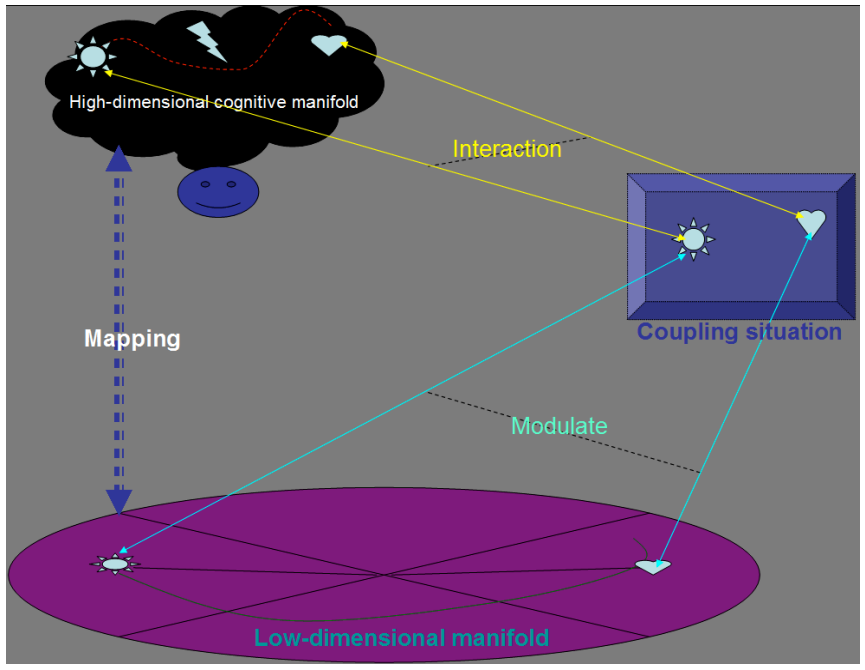

Fig. (3). Category-theory-based cognition observation in BSM. High-dimensional cognitive objects are induced by the coupling situation. The cognitive information is collected through manmachine interaction. Modulated by computers, the coupling situation can perform more collection interaction for the controllability of cognition observation.

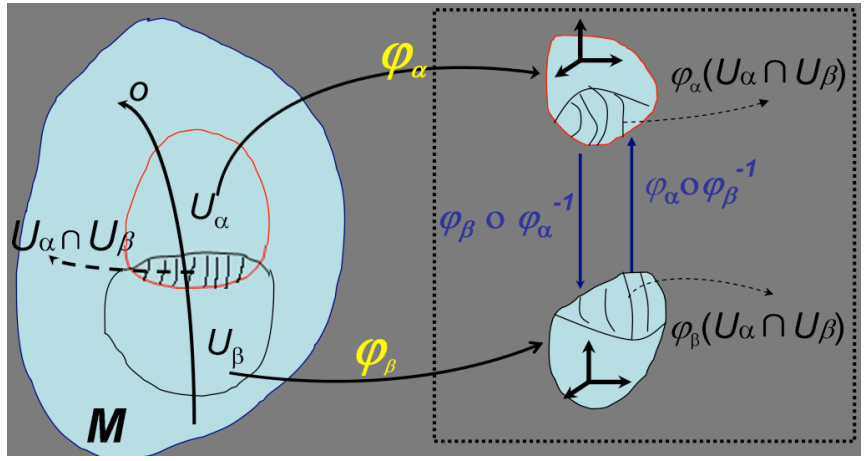

Fig. (4). An analysis of mapping a high-dimensional manifold to a metric space.

The measurement of o is described as following: (1) the domains where o goes through are mapped to a Euclidean space; (2) $\mathrm{U}_{\alpha} \cap \mathrm{U}_{\beta}=\mathrm{U}_{\alpha \beta}$ represents the intersection of two domains $\left(U_{\alpha}\right.$ and $\left.U_{\beta}\right)$, which is an important part of our investigation in cognitive manifolds because $U_{\alpha \beta}$ is the area where o must go through and be mapped to a metric space by two mapping methods; (3) a relationship between $\varphi_{\alpha}\left(U_{\alpha} \cap U_{\beta}\right)$ and $\varphi_{\beta}\left(U_{\alpha} \cap U_{\beta}\right)$ can be created with $U_{\alpha \beta}$ in the metric space. For example, two relationships (i.e. $\varphi_{\beta} O \varphi_{\alpha}{ }^{-1}$ and $\left.\varphi_{\beta} O \varphi_{\alpha}{ }^{-1}\right)$ can be created in separated parts of the metric space, which allows us to investigate the relationship between the two cognitive domains. Otherwise, the two domains cannot be studied in the metric space without their relationships; (4) the o can be studied by mapping to the metric space with the cognitive orbit and process.

For example, we can observe two cognitive objects $\mathrm{A}\left(\mathrm{x}_{1}\right.$, $\left.\mathrm{x}_{2}, \mathrm{x}_{3}\right)$ and $\mathrm{B}\left(\mathrm{x}_{1}, \mathrm{x}_{2}{ }_{2}, \mathrm{x}_{3}{ }_{3}\right)$ in BSM. Is there a relationship between $\mathrm{A}$ and $\mathrm{B}$ ? If the relationships are found between $\mathrm{x}_{1} \rightarrow \mathrm{x}^{\prime}{ }_{1}, \mathrm{x}_{2} \rightarrow \mathrm{x}_{2}{ }_{2}$ and $\mathrm{x}_{3} \rightarrow \mathrm{x}_{3}{ }_{3}$, is there a relationship between A and B? Fortunately, these mentioned relationships can be automatically analyzed by BSM.
We have found the potential ability of BSM as the cognition digitization and mathematical description. Additionally, the approaches in mathematics and physics can be introduced in the cognitive research in BSM.

In order to determine the coordinates of observed cognitive objects, according to manifold analysis, a coordinate system or a frame should be selected before mapping cognition to a Euclidean space.

In BSM, the situations can be described by content, operation and mathematics in a three-dimensional space. The content, operation and mathematics are called as the basic frame of situation [14]:

1) Content: cognitive objects and elements in the situation.

2) Operation: cognitive operations, logic and processes in the content of the situation.

3) Mathematics: quantities and properties of dynamics systems in the situation.

The content, operation and mathematical frame of the situation is called as COM frame (Fig. 5). The cognition frame varies greatly, and the alternative frame can be applied in the situations which show the individuality of cognition. The relationships among local structures of manifolds can be established based on the cognitive frame, which is an accurate and deep representation, a multi-layered abstraction and a concrete description of a local structure in a cognitive manifold.

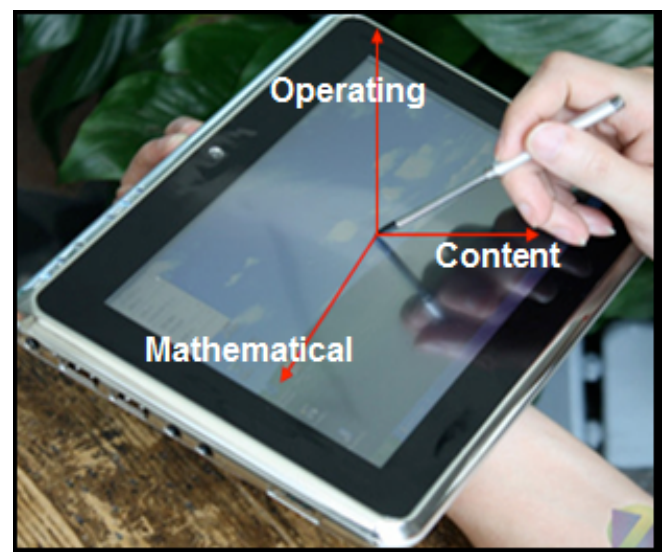

Fig. (5). COM cognition frame: content, operating and mathematical of situations

Based on the COM cognition frame, we can realize the information collection of cognitive operation in humanmachine situations, and transform them to cognitive symbol sequences, which can describe the processes of humanmachine interaction. The cognitive symbol sequence is the basis of cognition re-structuring, and the accuracy and validity of cognitive information relies on the design of the cognitive symbol sequence.

\subsection{Structure of BSM Low-dimensional Manifolds}

In mathematics, the manifolds can be approximated by graphs, or mapped to graphs. For graph $\mathrm{G}=(\mathrm{V}, \mathrm{E})$ which includes two sets, the $\mathrm{V}$ and $\mathrm{E}$ respectively denotes a set of vertices and edges. For the set $\mathrm{x}$ sampled from a $\mathrm{d}$ dimensional manifold, a one-to-one mapping between the 
data and vertices in $\mathrm{G}$ is established, and the similarity between a pair of samples is defined as an edge in G. Thus, a graph can be established utilizing these sample data. The graph and the corresponding manifold have a number of similar properties, among which the most important one is that both of them can be embedded in a Euclidean space. For the manifold, one corresponding graph is a topology object. and its topological properties can be represented by the edge weights. We apply the complex cognitive networks [15] to describe the cognitive manifolds, in which a node denotes a cognitive object and an edge denotes the logic relationship between nodes.

Currently, the complex network has became a popular technique that is not only used to describe the framework of open and complex systems in technology, biology and society, but also a powerful tool for topology and dynamic research in these fields. In the field of cognition and cognitive psychology represented by Piaget, the knowledge map in knowledge engineering, the concept map and the mind map are related to the complex network [16-19].

In BSM, the trees and networks are selected to present low-dimensional manifolds because they can be easily carried out by the computers. As an important non-linear structure, the nodes in the tree structure have multiple branches and are organized in multiple layers, which looks like the trees in the real world. There are plenty of objects organized in trees, such as the genealogy and organization of institutions. The tree structure is also widely applied in computer science. For example, the grammar structures of source codes can be presented as trees in compilation processes; the data can be organized as trees in database systems; the tree structure can be used to describe the execution of the algorithm in algorithm analysis.

The tree is similar with the network, and its structure can be easily transformed to the network structure. However, it is difficult to transform the network structure to the tree structure because there is no loop in trees. In this study, we introduce an attribute "link" in a node of a tree, which indicates a link between the current node and the one in another tree. With the link which is named as "virtual link", all topological networks can be presented as tree structures, and we name them "tree-based network" in this paper.

The "tree-based network" can unlimitedly extend, and its nodes can be generated in particular conditions. In BSM, if a finer-grained observation on a cognitive object is required, a sub-node of currently observed cognitive object will be generated (Fig. 6).

On the BSM cognitive observation platform, the objects and elements in high-dimensional cognitive manifolds are mapped to low-dimensional topological manifolds, and represented by the tree-based networks in the coupling situations. Similar with films in traditional photography, the treebased networks are the cognitive "films" for BSM observation.

First, we determined an observed object (i.e. obj). Then, a COM observation frame was created on obj in the coupling situations. When operations were performed on the obj by users, the cognitive symbol sequences were collected and imaged to the "films"(i.e. tree-based networks).

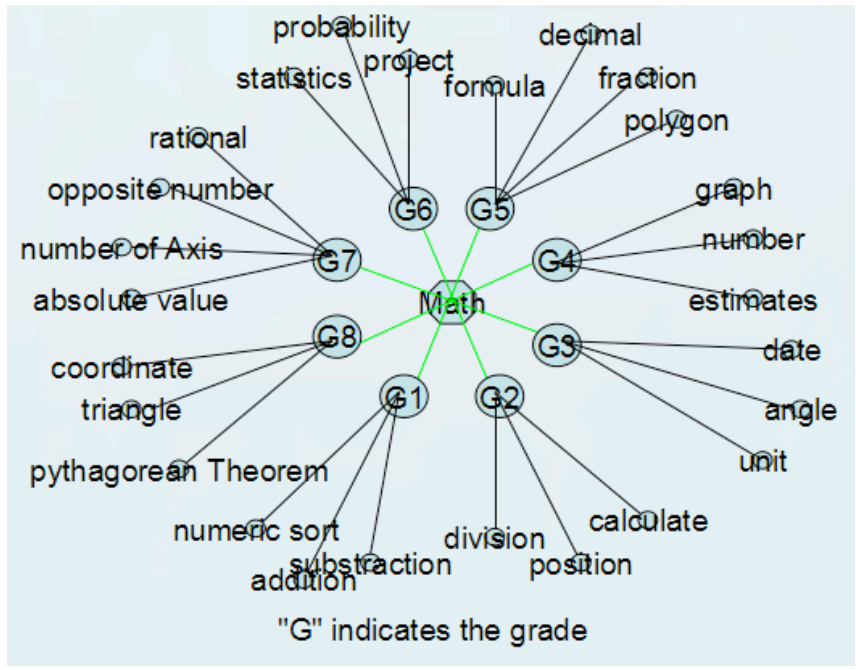

Fig. (6). A tree structure of knowledge in disciplines.

Based on the extensibility of the tree-based network and the feasibility of observation on the BSM platform, we can map a large amount of contents and structures in highdimensional cognitive manifolds to information manifolds for the digitization of cognitive structure, which is difficult to realize for the traditional cognitive psychology and pedagogy.

\subsection{Metrics of BSM Low-dimensional Manifolds}

Based on the topological structures of BSM lowdimensional manifolds, we created a cognition metric space by introducing metrics of cognition in topological manifolds. In mathematics, there are a number of powerful methods, such as calculus which can be used for analysis in a metric space. The objective in cognitive psychology is how to create a metric framework for cognition. Overall, our research framework can be described in Fig. (7).

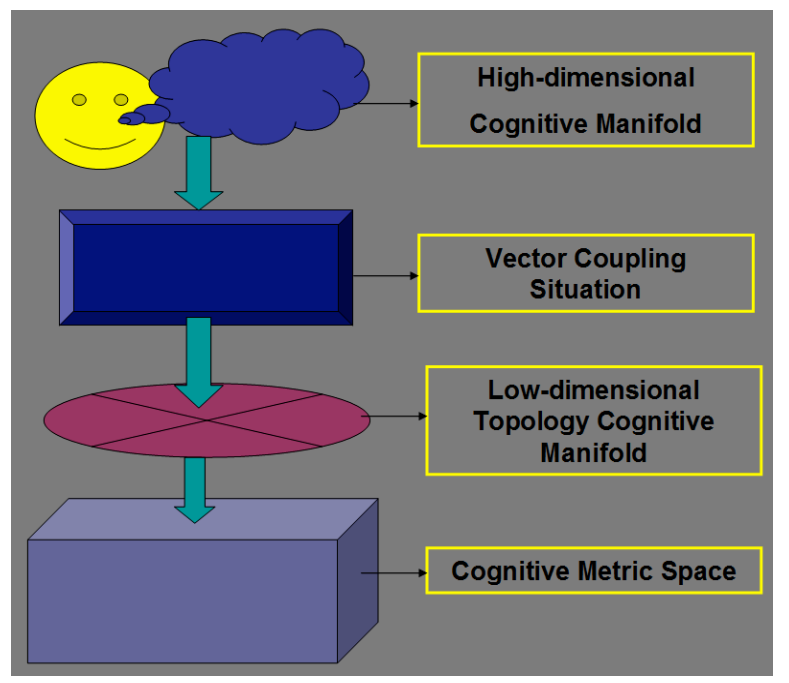

Fig. (7). A layered research framework of BSM cognition observation.

The methods of coordinate measurement in physics and mathematics can be introduced into the cognition measurement. And the "layer" is the most important characteristics of a cognition metric space. Similar with the tree-based networks, the cognitive content can be organized according to the layered topology (Fig. 8). 


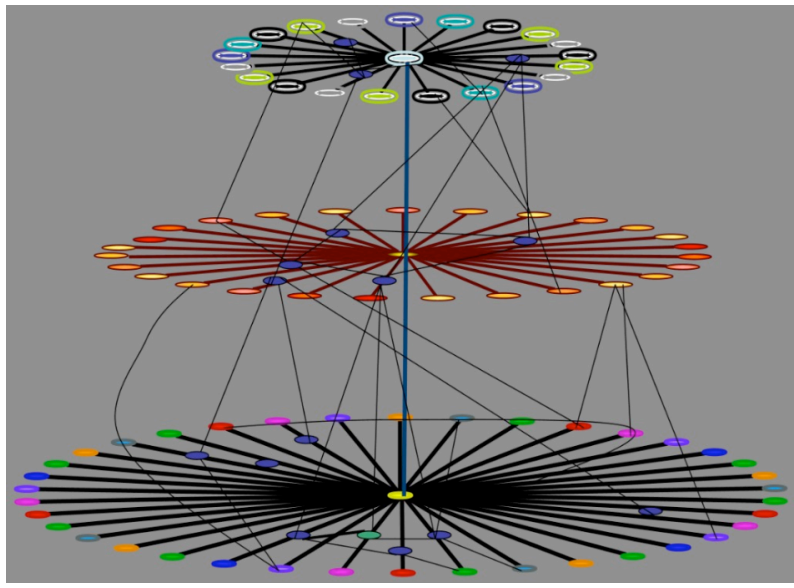

Fig. (8). A layered cognitive structure simple model.

\section{CASE STUDIES: VISUALIZATION OF BSM- BASED COGNITIVE STRUCTURES}

\subsection{Coupled Imaging of Cognitive Structures of Digital Games}

Taking the 24 Game played by students as an example, four digits (1-9) were generated by a computer, and students would try to figure out whether there was a method to get 24 by using a mixture of addition, subtraction, multiplication and division.

In the human-machine interaction system of the 24 Game, each calculation was collected as a record. Finally, we collected and visualized nearly 80 thousand of records from more than 2000 grade 3 to undergraduate students.

(1) Interface of collecting cognitive symbol sequences: four digits were randomly generated by the computer. As

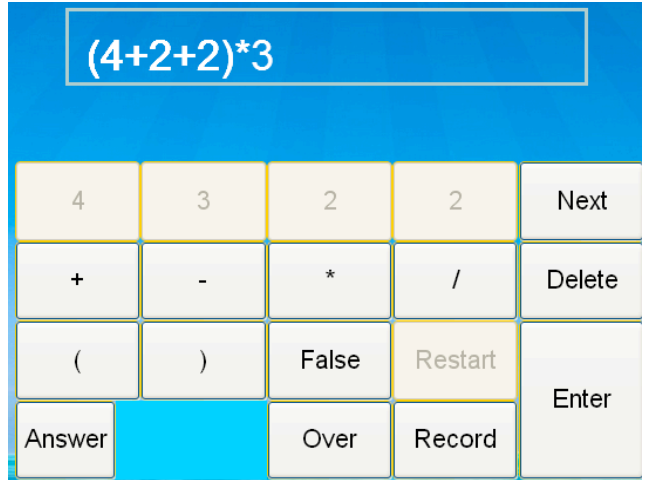

Fig. (9). Interface of collecting cognitive symbol sequences in 24 Game.

shown in Fig. (9), users provided an equation by choosing the corresponding symbols.

Through introduction and learning, the users could quickly understand the human-machine coupling situation, and complete the game by clicking and touching.

(2) Cognitive symbol sequence: when users clicked the cognitive sensors, the cognitive data including the user ID and time would be collected as shown in Fig. (10).

(3) Cognitive datasets: to process the large amount of cognitive symbol sequences, the basic tasks are to build the datasets based on their categories. For example, the data can be classified according to their average completion time to find out the records with high difficulty. Fig. (11) shows the game name, the number of testing times and the average completion time. The game "3-3-5-8" was tested for 81 times, and its average completion time is 15.1 seconds (Fig. 11).

$$
\begin{aligned}
& \mid(\mid(2)(2+\mid(2+4 \mid(2+4+\mid(2+4+2|(2+4+2)|(2+4+2) *|(2+4+2) * 3| \text { _true_spent: } 8 \mathrm{~s} \\
& \mid(\mid(2)(2+\mid(2+4 \mid(2+4+\mid(2+4+2|(2+4+2)|(2+4+2) *|(2+4+2) * 3| \text { true_spent: } 8 \mathrm{~s} \\
& \mid(2 \mid(2+\mid(2+4 \mid(2+4+\mid(2+4+2|(2+4+2)|(2+4+2) *|(2+4+2) * 3| \text { true_spent: } 8 \mathrm{~s} \\
& |3| 3 * \mid 3 *(|3 *(4)| 3 *(4+\mid 3 *(4+2 \mid 3 *(4+2+\mid 3 *(4+2+2|3 *(4+2+2)| \text { true_spent: } 11 \mathrm{~s} \\
& |3| 3 * \mid 3 *(\mid 3 *(4) 3 *(4+\mid 3 *(4+2 \mid 3 *(4+2+\mid 3 *(4+2+2|3 *(4+2+2)| \text { true_spent: } 11 \mathrm{~s} \\
& |3||<|(4 \mid(4+\mid(4+2 \mid(4+2 * \mid(4+2 * 2|(4+2 * 2)|(4+2 * 2) *|(4+2 * 2) * 3| \text { true_spent:9s } \\
& |3| 3 * \mid 3 *(\mid 3 *(2 \mid 3 *(2 * \mid 3 *(2 * 2 \mid 3 *(2 * 2+\mid 3 *(2 * 2+4|3 *(2 * 2+4)| \text { true_spent: } 65 \text { s } \\
& \mid(|(4)|(4+\mid(4+2 \mid(4+2+\mid(4+2+2|(4+2+2)|(4+2+2) *|(4+2+2) * 3| \text { _true_spent: } 8 \mathrm{~s}
\end{aligned}
$$

Fig. (10). Cognitive symbol sequences in 24 game.

\begin{tabular}{l|c|c}
\hline \hline $3-3-5-8$ & 81 & 15.1 \\
\hline $2-3-7-8$ & 83 & 15.1 \\
\hline $2-4-4-5$ & 41 & 15.0 \\
\hline $7-8-8-9$ & 15 & 14.9 \\
\hline $6-6-6-9$ & 14 & 14.9 \\
\hline $1-4-6-6$ & 50 & 14.7 \\
\hline $3-4-9-9$ & 50 & 14.7 \\
\hline $2-4-4-9$ & 34 & 14.6 \\
\hline $2-3-6-6$ & 58 & 14.4 \\
\hline $2-2-8-8$ & 27 & 14.4 \\
\hline $2-6-6-9$ & 25 & 14.3 \\
\hline $1-6-7-8$ & 174 & 14.3 \\
\hline
\end{tabular}

Fig. (11). Average completion time of 24 game. 
(4) Cognitive topological analysis: the cognitive topological space was created based on the cognitive datasets. For example, we used an algebraic method to topologize $(4+2+2) * 3$ to $(\mathrm{A}+\mathrm{B}+\mathrm{C}) * \mathrm{D}$. Thus, all cognitive records (i.e. coreness), such as $(3+3+2) * 3$ and $(1+3+2) * 4$, were mapped to the image of $(\mathrm{A}+\mathrm{B}+\mathrm{C}) * \mathrm{D}$, and a new layered cognitive topological space was created which was named as "quotient topological space".

We obtained 232 images from 62401 records by algebraic analysis. This number is much smaller than that generated by the combination methods, indicating that the cognitive operations and the pure mathematical methods were different. The mentioned coreness can be called as the cognitive computation genes of the 24 game, as shown in Fig. (12).

(5) Imaging of cognitive metrics: the four edges (Q1, Q2, Q3 and Q4) of a given quadrilateral can respectively represent the values of the four digits in 24 game. Each cognitive computation in the 24 Game was mapped to a point on an edge of the quadrilateral, and the connection of these points is shown in Fig. (6). The completion time of the cognitive computation can be marked in colors on the points. In Fig. (13), the red and green colors indicate the longest and the shortest completion times, respectively.

Based on the human-machine interaction, we analyzed the cognitive computation in 24 Game from the perspective of processes, topology and dynamics. Moreover, we visualized the abstract data to support the education application.

\subsection{Analysis of Cognitive Structures based on Tests}

On BSM platform, we collected the data from study and testing, and analyzed the understanding status of students using the methods as described above. Our analysis consists of the steps as following:

(1) Test vectorization: we scanned the test papers and collected the answers for all questions.

\begin{tabular}{|l|l|l|}
\hline$\left(\left(A^{*} B\right)-C\right) / D$ & $((A+B)-C))^{*} D$ & $((A-B)-C)^{*} D$ \\
\hline$((A+B)-C)^{*} D$ & $((A-(B-C)))^{*} D$ & $((A-B) / C)^{*} D$ \\
\hline$((A+B) / C)^{*} D$ & $((A-B))^{*}(C+D)$ & $((A-B+C))^{*} D$ \\
\hline$((A+B+C))^{*} D$ & $((A-B))^{*} C * D$ & $((A / B)+C){ }^{*} D$ \\
\hline
\end{tabular}

Fig. (12). Partial patterns.

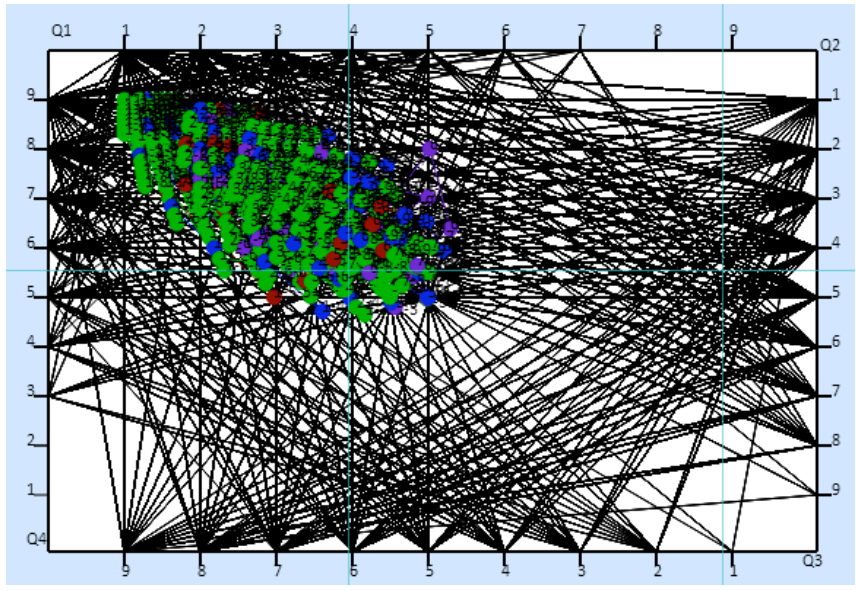

Fig. (13). Imaging of cognitive topology.

Taking the question $3+9=$ ? as an example, the students were required to input the answers in a pre-defined manner. Then, computers could easily collect the answers as the testing data (Fig. 14).

(2) Analysis of testing points and knowledge structures: according to the educational objectives, the testing points in the test paper were abstracted to a knowledge structure, which was a layer above the test paper. Various structures would be given by professors, indicating their different understandings of the tests (Fig. 15).

(3) Analysis of knowledge structure and discipline structure: after (1) test vectorization and (2) knowledge structure

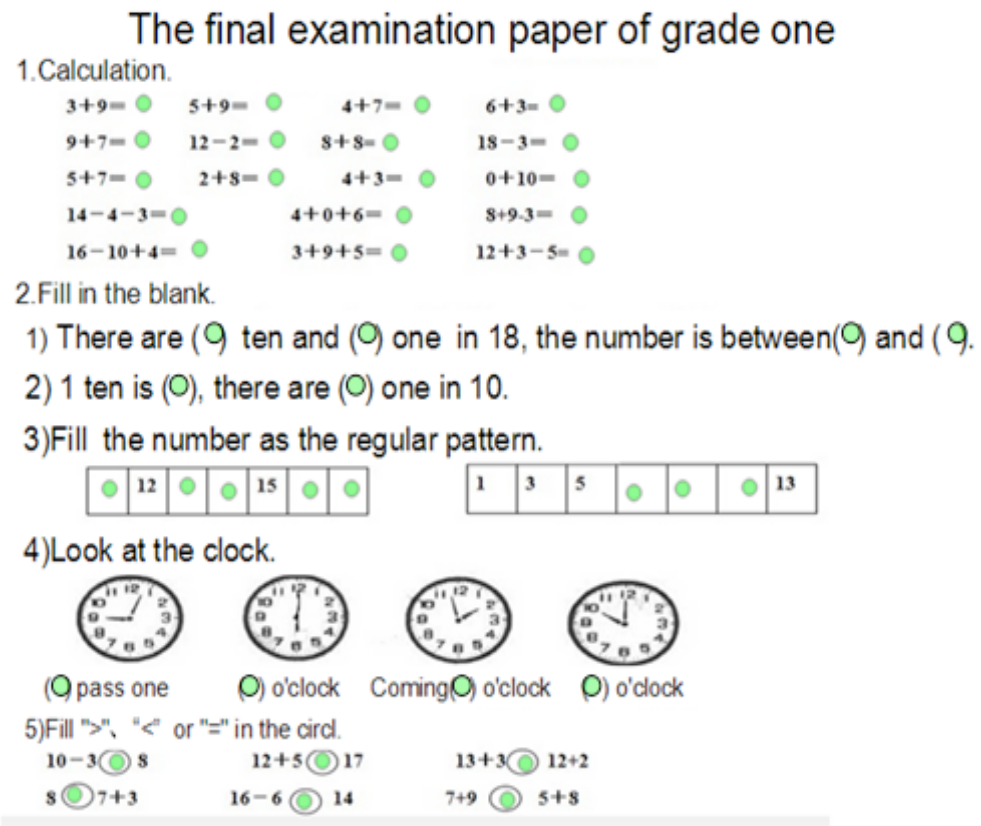

Fig. (14). Test paper vectorization and answer collection. 
The final examination paper of grade one

1. Calculation

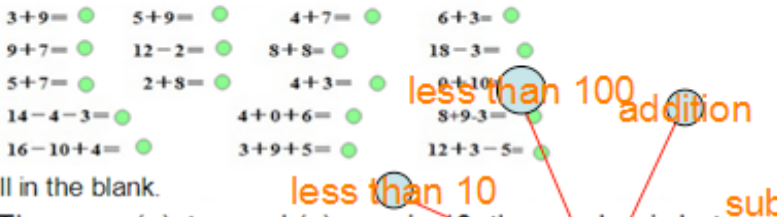

1) There are (0) ten and $(0)$ one in 18 , the number is between

2) 1 ten is $(0)$, there are $(0)$ one in 10 .

3)Fill the number as the regular pattern

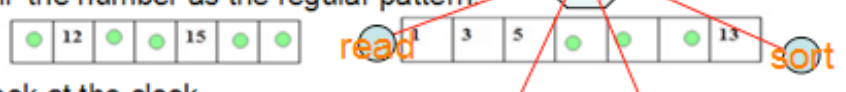

4)Look at the clock.

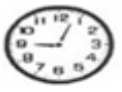

()) pass one o'clock

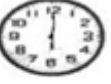

0) o'clock Comingo) o'clock O) o'clock figure

5)Fill " $>"$ " "< or " $="$ in the circl.

$\begin{array}{lrr}10-3 \bigcirc 8 & 12+5 \bigcirc 17 & 13+3 \bigcirc 12+2 \\ s \bigcirc 7+3 & 16-6 \bigcirc 14 & 7+9 \bigcirc 5+8\end{array}$

Fig. (15). Knowledge structure in the test paper.

The final examination paper of grade one 1.Calculation

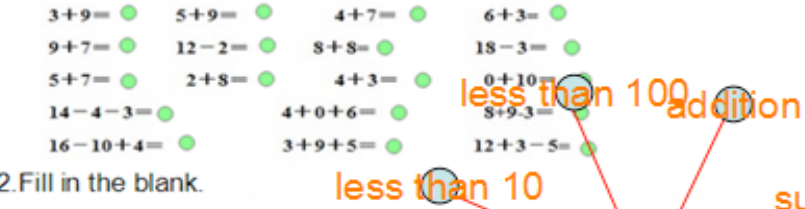

1) There are $(0)$ ten and $(0)$ one in 18 , the number is between(o) and $(0)$

2) 1 ten is $(Q)$, there are $(Q)$ one in 10.

3)Fill the number as the regolar pattern

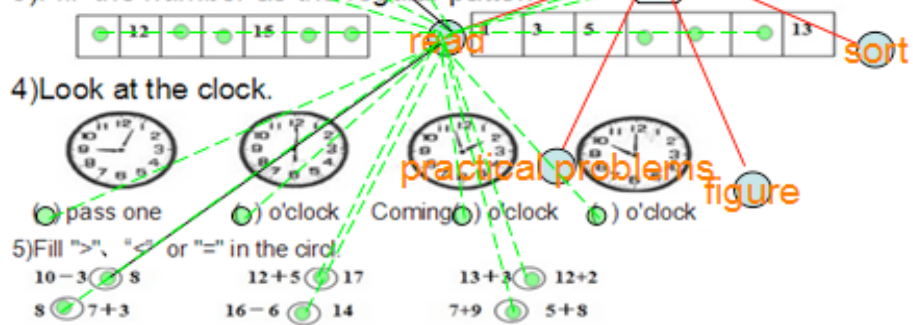

Fig. (16). The process of associating the knowledge structure and testing points.

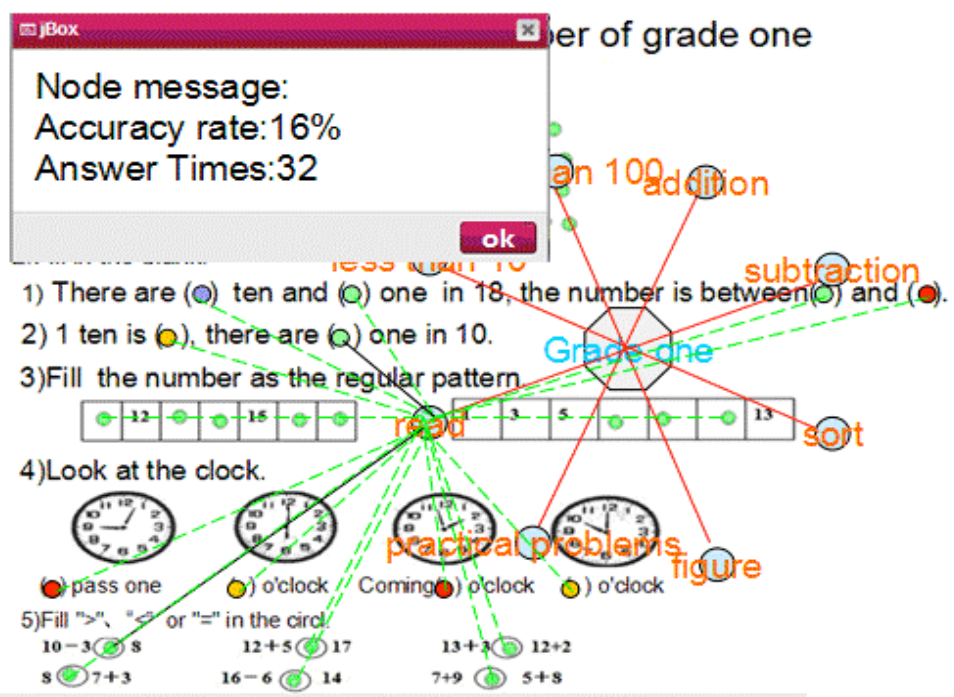

Fig. (17). Automatically analyze the results of testing points. 
determination, we associated the testing points with the knowledge structure. Undoubtedly, alternative testing point would be associated with different knowledge, and alternative knowledge would also be associated with different knowledge (Fig. 16).

(4) Automatic data collection from test papers: after associating the knowledge structure with the testing points, we applied the automatic analysis to collect answers from testing points. For example, we automatically obtained the rates of correct/wrong of testing points, and marked them in red, yellow and green colors to indicate the understanding status of students (Fig. 17).

(5) the discipline structure and ability analysis of students: utilizing the tree-based structure of knowledge, we can further analyze the cognitive ability, and associate them with the knowledge analysis in the tests.

We have demonstrated the tree-based analysis method in BSM digital environments by collecting behavior information in tests and understanding the status of knowledge structures. Additionally, we observed and analyzed the status of cognitive structures, and provided the evidence and experience to empirical research and automation for education.

\section{CONCLUSION}

From the point of view of the advanced cognitive science and category theory, the human-machine cognitive coupling environment becomes the advanced observation platform for cognition and learning activities, as "Brain cognition body cognitive coupling Situations-information Manifolds" (BSM).

Based on the topological manifolds analysis, the treebased networks were selected to represent the topological structures of low-dimensional cognitive manifolds. We restructured the observed cognitive data, and created a layered and vectorized cognitive observation space.

Utilizing the model of layered and vectorized cognitive structures in human-machine learning environments, we can establish an automatic learning environment, and provide the empirical information of cognitive structures for education. This method can also support the cognitive dynamics research.

\section{CONFLICT OF INTEREST}

The authors confirm that this article content has no conflict of interest.

\section{ACKNOWLEDGEMENTS}

The paper is supported by the following funds: the Education Ministry Fund in Fujian Province (JA12321,
JA13318) and the Key Scientific Project Fund in Nanping, Fujian (N2012Z06-7), Wuyi University Research Fund for Young Teachers (xq201025).

\section{REFERENCES}

[1] S. Meng, R. Cheng, "Cognitive coupling states based on tree cognitive fields," In: International Conference on Computer Communication and Management, Singapore, 2011, pp. 593-597.

[2] S. Meng, R. Cheng, Y. Liu, "Cognitive topology mapping based on human-computer interaction," Journal of Internet Technology and Secured Transactions, vol. 1, pp.123-130, 2012.

[3] J. Hollan, E. Hutchins, D. Kirsh, "Distributed cognition: toward a new foundation for human-computer interaction research," $A C M$ Transactions on Computer-Human Interaction, vol. 7, pp. 174-196, 2000 .

[4] http://hci.ucsd.edu[EB/OL]

[5] A. Clark, Supersizing The Mind, Oxford University Press, 2008, pp. $3-43$.

[6] S. Meng, C. Liu, W. Yu, "Cognitive digital manifold based on CHI," Computer and Information Technology, vol. 21, pp.1-8,49, 2013.

[7] S. Yang, L. Guo, W. Yu, C. Liu, S. Meng, "Learning dynamics analysis based on computer-human interaction," International Journal of Emerging Technologies in Learning, vol. 8, no. 5, pp. 32-36, 2013.

[8] Y. Liu, S. Meng, R. van der Zwan, "The logical framework of 1:1 digital learning, teachers talk about what's important," In: International Teacher Education Dialogue Conference, pp. 88-93, 2012.

[9] T. J. Van Gelder, "Dynamic approaches to cognition," The MIT Encyclopedia of cognitive sciences, pp. 244-246, 1999.

[10] R. M. French, E. Thomas, "The dynamical hypothesis in cognitive science: a review essay of mind as motion, "Minds and Machines, vol. 11, no. 1, pp. 101-111, 2001

[11] S. Yang, W. Yu, C. Liu, R. Cheng, L. Guo, S. Meng, "A cognitive topology structure observation approach based on man-machine coupling states," Computer Modelling and New Technologies, vol. 17, no. 4, pp. 174-184, 2013.

[12] S. Meng, Z. Li, H. Jiang, L. Qiu, "Complex word network model based on cognitive coupling states," In: International Conference on Information Society, London, United Kingdom, 2012.

[13] J. Piyajie, G. Henriques, E. Ascher, T. Brown, Morphism and category: comparison and transformation, Shanghai: East China Normal University Press, September, 2005, 11-37.

[14] S. Yang, C. Liu, R. Cheng, "Vectorization methods of HCI situations based on cognitive frame," Journal of Chongqing University of Technology (Natural Science), vol. 27, pp.102-106, 2013.

[15] S. Meng, "Visualization complex cognitive networks, "IPEDR, vol. 27, pp. 1-5, 2012

[16] J. T. Cacioppo, W. von Hippel, J. M. Ernst, "Mapping cognitive structures and processes through verbal content: the thought-listing technique," Journal of Consulting and Clinical Psychology, vol. 65, pp. 928-940, 1997.

[17] B. Yoram, "When the need for cognitive structure does not cause heuristic thinking: the moderating effect of the perceived ability to achieve cognitive structure," Psychology, vol. 1, pp. 96-105, 2010.

[18] D. M. Dimitrov, T. Raykov, "Validation of cognitive structures: a structural equation modeling approach," Multivariate Behavioral Research, vol. 38, no. 1, pp. 1-23, 2003.

[19] T. Froese, K. Suzuki, Y. Ogai, T. Ikegami, "Using human-computer interfaces to investigate 'mind-as-it-could-be' from the first-person perspective, "Cognitive Computation, vol. 4, pp. 365-382, 2012.

\footnotetext{
Received: September 22, 2014

Revised: November 30, 2014

Accepted: December 02, 2014

(C) Rengui et al.; Licensee Bentham Open.
}

This is an open access article licensed under the terms of the Creative Commons Attribution Non-Commercial License (http://creativecommons.org/licenses/by-nc/3.0/) which permits unrestricted, non-commercial use, distribution and reproduction in any medium, provided the work is properly cited. 